\title{
Erratum: Cosmic expansion in extended quasidilaton massive gravity [Phys. Rev. D 91, 041301(R) (2015)]
}

Tina Kahniashviliø, Arjun Kar, George Lavrelashvili, Nishant Agarwal, Lavinia Heisenberg, and Arthur Kosowsky

Q (Received 1 October 2019; published 21 October 2019)

DOI: $10.1103 /$ PhysRevD.100.089902

In this paper, Eq. (7) is missing a variation of the $\alpha_{\sigma}$ term in the action. This restricts our parameter space to $\dot{\phi}^{0}(t) / n(t)=$ constant and the late-time solution corresponds to $J \rightarrow 0$. The correct constraint equation is

$$
m_{g}^{2} q \frac{X}{H+\dot{X} / X}\left[4(X-1) J H+\frac{\dot{X}}{X}\left\{3 X\left(\alpha_{3}(X-1)-2\right)+J(4 X-1)\right\}\right]-\frac{\alpha_{\sigma}}{a^{4} X} \frac{\mathrm{d}}{\mathrm{d} t}\left[\frac{a^{4}}{q}\left(H+\frac{\dot{X}}{X}\right)(X-1) J\right]=0 .
$$

The corresponding quasidilaton equation of motion now matches that given in [1], Eq. (22). The conclusions of this paper have not changed.

We thank Emir Gümrükçüoğlu for pointing out this error.

[1] A. E. Gumrukcuoglu, K. Koyama, and S. Mukohyama, Phys. Rev. D 94, 123510 (2016). 